\title{
Desmopressin in the treatment of daytime urinary frequency in patients with multiple sclerosis
}

\author{
Patricia A Hoverd, Clare J Fowler
}

\begin{abstract}
Twenty two patients with multiple sclerosis, complaining of frequency of day time micturition, completed a double blind crossover trial of desmopressin (DDAVP nasal spray) versus placebo. There was a significant decrease in micturition frequency in the 6 hour post-treatment period from 3.1 voids after placebo to 2.4 voids and a significant reduction in urinary volume after desmopressin. Eighty per cent of patients preferred the active treatment phase. Mean 24 hour urinary volume did not differ between active and placebo treatments and patients did not complain of increased night time frequency. Transient symptoms of hyponatraemia occurred in one patient but these resolved within 48 hours of stopping desmopressin. There were otherwise no side effects and mean serum sodium concentrations of the group remained unchanged throughout the study. The clinical indications for prescribing daytime desmopressin are discussed and the importance of patient compliance stressed.

(f Neurol Neurosurg Psychiatry 1998;65:778-780)
\end{abstract}

Keywords: multiple sclerosis; bladder dysfunction; urinary frequency; desmopressin

Department of

Uro-Neurology,

National Hospital for

Neurology and

Neurosurgery, Queen

Square, London, UK

P A Hoverd

C J Fowler

Correspondence to: Dr Clare J Fowler,

Department of

Uro-Neurology, National

Hospital for Neurology and

Neurosurgery, Queen

Square, London WC1N

3BG, UK. Telephone 0044

1718373611 extension

3418; fax 0044171813

4587; email

c.fowler@ion.ucl.ac.uk

Received 19 February 1998 and in revised form

25 May 1998

Accepted 1 June 1998
Desmopressin is a synthetic vasopressin analogue with a duration of action of 9.5-13 hours. First introduced for the treatment of neurogenic diabetes insipidus it was then shown to be effective in the management of primary nocturnal enuresis. Several studies then showed it to be effective in managing nocturia in patients with multiple sclerosis. ${ }^{1-3}$ However, daytime urgency and frequency is also often highly distressing for patients with multiple sclerosis, seriously disrupting work and social activities. We found that many of the patients with multiple sclerosis given desmopressin to lessen night frequency with benefit, admitted on direct questioning that they had occasionally taken it during the day instead.

This study was to examine the effect on voiding frequency of desmopressin compared with placebo, the effect of desmopressin on 24 hour urine production, and the safety and tolerance of desmopressin in patients with multiple sclerosis.

\section{Patients and methods}

Twenty eight patients with multiple sclerosis and neurogenic bladder dysfunction were screened, and 26 (10 men, 16 women) recruited into the study. Their ages ranged from 18-65 years with a mean age of 43 . For patients to be able to collect their urine and measure the volume it was necessary that they should have sufficient lower limb power to stand and also that they should be cognitively unimpaired. The inclusion criterion was that a patient had eight or more episodes of voiding a day. Seventeen of the patients were already on treatment for neurogenic incontinence including full dosages of anticholinergic therapy and intermittent catheterisation if they had been shown to have incomplete emptying. Patients with diabetes, heart disease, hypertension, or renal disease or those taking diuretic therapy were excluded

Four patients were withdrawn from the study, three for various reasons before starting the treatment phases. One patient developed headache and other symptoms of fluid retention and was found to be hyponatraemic after active treatment and was withdrawn. None of these four patients were subsequently considered in the analysis

Desmopressin was given from a nasal spray providing metered doses of $10 \mu \mathrm{g}$ per spray and the patients were instructed to use one spray in each nostril at the same time each day between 800 am and $200 \mathrm{pm}$.

The study took place over 6 weeks. Patients were assigned to placebo or desmopressin treatment in random order. The first 2 weeks were a "run in" phase during which they practised handling the spray bottle and filling in diary cards recording frequency of micturition as well as amounts voided. Baseline blood pressure was recorded together with weight and blood sodium concentrations. At the beginning of the $3 \mathrm{rd}$ week observations were repeated and bottle A was given. Patients continued to record voiding frequency and had to use the spray at the same time each day. Two weeks later, at the beginning of the 5 th week, bottle A was replaced by bottle B. On the final day of the study blood pressure, weight, and blood sodium were measured again and 
Urinary variables during placebo and desmopressin treatment

\begin{tabular}{|c|c|c|c|c|}
\hline & Run in & Placebo & Desmopressin & $\begin{array}{l}\text { p Value } \\
\text { (placebo/ } \\
\text { desmopressin) }\end{array}$ \\
\hline \multicolumn{5}{|c|}{$\begin{array}{l}\text { Number of voids in } 6 \text { hours } \\
\text { (after treatment where applicable): }\end{array}$} \\
\hline Mean & 3.2 & 3.1 & 2.4 & \multirow{2}{*}{$0.008^{\star}$} \\
\hline SD & 1.3 & 1.4 & 0.9 & \\
\hline \multicolumn{5}{|c|}{$\begin{array}{l}\text { Mean urine volume in } 6 \text { hours } \\
\text { (after treatment where applicable): }\end{array}$} \\
\hline Mean & 384 & 342 & 246 & \multirow[t]{2}{*}{$0.006^{\star}$} \\
\hline $\mathrm{SD}$ & 166 & 166 & 99 & \\
\hline \multicolumn{5}{|c|}{ Mean 24 hours urine volume $(\mathrm{ml})$ : } \\
\hline Mean & 1290 & 1272 & 1218 & \multirow[t]{2}{*}{0.052} \\
\hline $\mathrm{SD}$ & 436 & 482 & 455 & \\
\hline \multicolumn{5}{|c|}{ Number of night time voids: } \\
\hline Mean & 1.2 & 1.4 & 1.5 & \multirow[t]{2}{*}{0.263} \\
\hline SD & 1.2 & 1.1 & 1.2 & \\
\hline \multicolumn{5}{|c|}{ Serum sodium (mmol/1): } \\
\hline Mean & 139.9 & 139.7 & 139.2 & \\
\hline SD & 3.0 & 3.0 & 3.3 & \\
\hline
\end{tabular}

${ }^{\star}$ Significant difference $(\mathrm{p}<0.05)$.

patients were asked which study period treatment they had preferred.

Results were analysed using ANOVA for crossover designs.

\section{Results}

Micturition frequency measured during the 6 hours after the use of the spray decreased significantly $(p<0.05)$ during the period of treatment with desmopressin to a mean of 2.4 voids, compared with both the run in period of 3.2 voids and placebo treatment 3.1 voids (table). There was a significant reduction $(\mathrm{p}<0.05)$ in mean urine volume produced during the active treatment period to $246 \mathrm{ml}$ from $384 \mathrm{ml}$ and $342 \mathrm{ml}$ during the run in and placebo periods respectively. The mean 24 hour urine volume did not differ significantly between the different periods, nor was there an increase in night time voiding frequency. Analysis of the preference data showed that $80 \%$ preferred the active to the placebo period of treatment, presumably because of the lessening of frequency of micturition.

The patient who was withdrawn on becoming hyponatraemic complained of general malaise, headache, and nausea and was found to have a serum sodium of $134 \mathrm{mmol} / \mathrm{l}$. The symptoms resolved within 48 hours of discontinuing treatment and the blood sodium concentration returned to $141 \mathrm{mmol} /$ when checked a week later. There was no significant change in blood pressure during this period.

\section{Discussion}

In this study the effect of desmopressin has been shown to be only on the voiding frequency and urine volume in the 6 hour period after use of the medication. No other measurements differed between the run in period, placebo treatment, and active treatment. In particular there was no compensatory night time frequency nor evidence of water retention as the 24 hour urine volumes were the same in all three periods. The design and results of this study are almost identical to those reported by Fredrikson ${ }^{4}$ who also found the only significant change to be a reduction in number of voids in the 6 hours after the active treatment phrase (from 3.1 to 2.6). Our study found a somewhat higher patient preference for the active treatment phase- $80 \%$ compared with Fredrikson's ${ }^{4} 68 \%$. A placebo controlled trial study using oral desmopressin in 13 patients with multiple sclerosis and daytime urinary symptoms also showed a reduction in micturition frequency in the 6 hour period after active treatment. ${ }^{5}$

From these three studies there is conclusive evidence that patients with multiple sclerosis and urinary frequency and urgency can be safely provided with a period of some hours when urine production is reduced and voiding is lessened after the administration of desmopressin. This can be of considerable advantage when undertaking a long journey or attending a meeting or social occasion when access to a suitable toilet may be difficult.

The concern is the development of hyponatraemia. In our study one patient developed this problem but in a study of desmopressin in the management of night time frequency in patients with multiple sclerosis, four out of 17 patients developed symptomatic and biochemical hyponatraemia. ${ }^{3}$ The authors speculated that some patients with multiple sclerosis might have subtle hypothalamic defects in antidiuretic hormone control making them particularly susceptible to hyponatraemia. However in that study, as in ours the mean serum sodium concentration of the group did not change after desmopressin, suggesting that the development of hyponatraemia is an idiosyncratic response. How can this finding best be used to guide clinical practice? It is often not convenient to get the patient back 1 week after starting treatments to measure the sodium and a reasonable effective alternative is to warn patients clearly what the possible symptoms of hyponatraemia might benamely, malaise, headache, and nausea-and to discontinue using desmopressin and contact their doctor should they develop any such complaints. A measurement of serum sodium can then be made and the patient advised to avoid the use of desmopressin in future if hyponatraemia is demonstrated.

The other question that arises is, when should desmopressin be prescribed to patients with multiple sclerosis and troublesome urinary symptoms? A patient with such disabling symptoms might reasonably be expected to be given any effective treatment and be unimpressed by the argument that their urinary symptoms were due to incomplete bladder emptying and detrusor hyperreflexia and therefore better treated by regular intermittent catheterisation and anticholinergic medication. ${ }^{6}$ In the author's practice, use of desmopressin is reserved for those already using first line therapy but who are occasionally in need of additional measures. It is prescribed to them after a strict warning about the possible risk of hyponatraemia and its symptoms and instructions that it must not be used more than once in 24 hours. It therefore seems reasonable to avoid prescribing it for patients in whom there is doubt about their possible compliance such as those with cognitive impairment. It should 
probably also be avoided in those with severe immobility and dependent oedema.

We acknowledge the financial assistance received from Ferring Pharmaceuticals UK, which paid the salary of PH. The suppor of Dr Brian Donovan in particular is gratefully acknowledged.

1 Hilton P, Hertogs K, Stanton S. The use of desmopressin DDAVP) for nocturia in women with multiple sclerosis. $f$ Neurol Neurosurg Psychiatry 1983;46:854-5.

2 Eckford S, Swami K, Jackson S, et al. Desmopressin in the treatment of nocturia and enuresis in patients with multiple sclerosis. Br f Urol 1994;74:733-5.
3 Valiquette G, Herbert J, Meade-D'Alisera P. Desmopressin in the management of nocturia in patients with multiple sclerosis. Arch Neurol 1996;53:1270-5.

4 Fredrikson S. Nasal spray desmopressin treatment of bladder dysfunction in patients with multiple sclerosis. Acta Neuro Scand 1996;94:31-4.

5 Kinn A-C, Larsson P. Desmopressin: a new principle for symptomatic treatment of urgency and incontinence in patients with multiple sclerosis. Scand f Urol Nephrol 1990; 24:109-12.

6 Fowler CJ, van Kerrebroeck PEV, Nordenbo A, et al. Treatment of lower urinary tract dysfunction in patients with multiple sclerosis. F Neurol Neurosurg Psychiatry 1992;55: 986-9. 